\title{
A SIMHEURISTIC FOR THE UNMANNED AERIAL VEHICLE SURVEILLANCE-ROUTING PROBLEM WITH STOCHASTIC TRAVEL TIMES AND RELIABILITY CONSIDERATIONS
}

\author{
Javier Panadero \\ Angel A. Juan \\ Alfons Freixes \\ Universitat Oberta de Catalunya - IN3 \\ Euncet Business School \\ Carl Friedrich Gauss Av. \\ Castelldefels, 08860, SPAIN
}

\author{
Carles Serrat \\ Manel Grifoll \\ Universitat Politècnica de Catalunya \\ Dept. of Mathematics - EPSEB \\ Dept. of Civil and Environmental Eng. \\ Barcelona, 08028, SPAIN
}

Mohammad Dehghanimohamamdabadi

Northeastern University

Dept. of Mechanical and Industrial Engineering

360 Huntington Avenue

Boston, MA 02115, USA

\begin{abstract}
In the unmanned aerial vehicle (UAV) surveillance-routing problem, a limited fleet of UAVs with drivingrange limitations have to visit a series of target zones in order to complete a monitoring operation. This operation typically involves taking images and / or registering some key performance indicators. Whenever this surveillance action is repetitive, a natural goal to achieve is to complete each cycle of visits as fast as possible, so that the number of times each target zone is visited during a time interval is maximized. Since many factors might influence travel times, they are modeled as random variables. Reliability issues are also considered, since random travel times might prevent a route from being successfully completed before the UAV runs out of battery. In order to solve this stochastic optimization problem, a simheuristic algorithm is proposed. Computational experiments contribute to illustrate these concepts and to test the quality of our approach.
\end{abstract}

\section{INTRODUCTION}

In the context of this paper, the concept of surveillance refers to the monitoring of a set of geographic areas with the purpose of detecting the occurrence of occasional events, which are often unannounced. Examples of such occasional events could be: (i) the potential occurrence of violent incidents in large urban areas, which require regular inspection by police patrols; (ii) the presence of migrants crossing the sea on fragile boats and assuming a high risk for their lives; (iii) the existence of refugees, escaping from war zones, who might need humanitarian assistance; (iv) the presence of potentially dangerous oil vessels or nuclear submarines traveling along the coast; $(v)$ the sudden emergence of wildfires in protected or peri-urban forest areas, specially during periods of hot and dry weather; or (vi) the detection of suspicious people in residential areas to mitigate the risk robbery and other criminal activities. We will assume that this monitoring process will be carried out via the use of unmanned aerial vehicles (UAVs). 


\section{Panadero, Juan, Serrat, Grifoll, Dehghanimohamamdabadi, and Freixes}

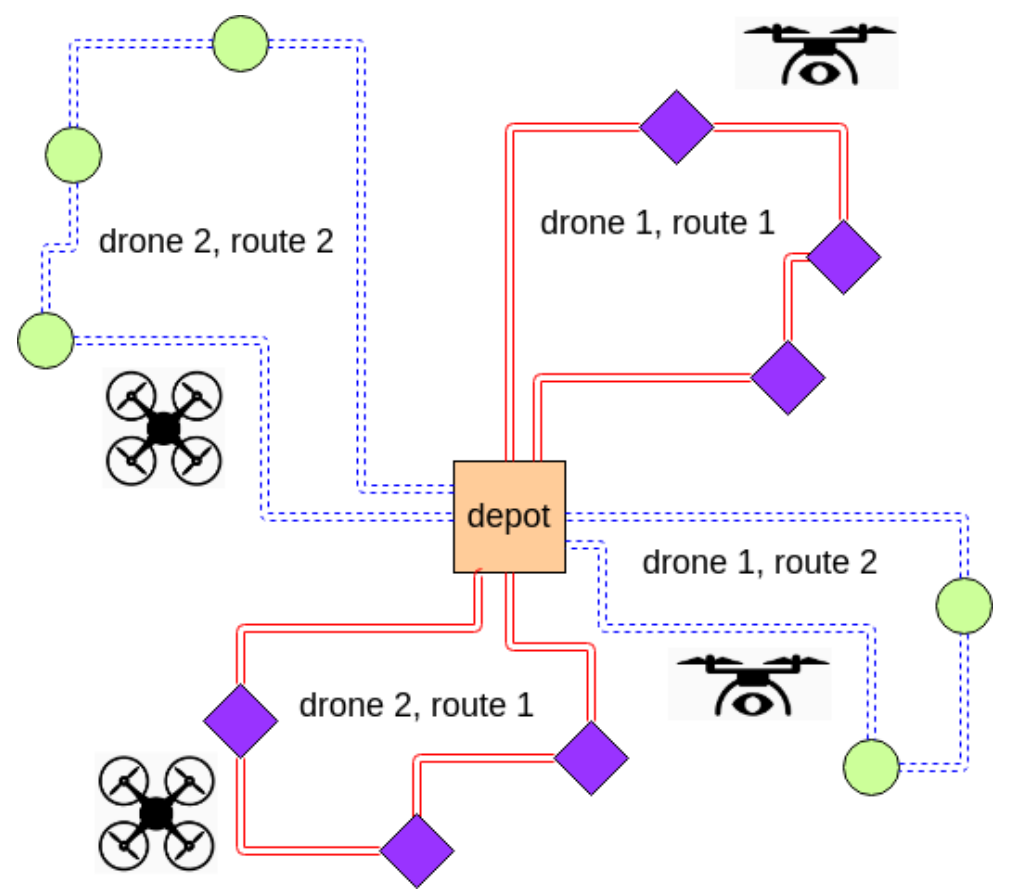

Figure 1: A simple example of the surveillance VRP with two UAVs and two routes each.

This work focuses on tactical and operational issues related to surveillance activities. In particular, it analyzes a variant of the well-known vehicle routing problem (VRP) (Faulin et al. 2008; Faulin and Juan 2008; Juan et al. 2009). In the considered VRP, a limited fleet of UAVs initially located at a depot are employed to visit a series of target zones in order to monitor them. The monitoring operation typically involves taking images of each target zone and / or registering some key performance indicators (e.g., temperature, humidity, etc.). Whenever this operation is cyclically repeated, the term persistent surveillance is employed, and one logical goal to achieve is to complete each cycle of visits as fast as possible. Figure 1 shows a simple example of a surveillance VRP where two UAVs have to complete a total of four monitoring routes. Thus, each UAV has to complete the first route assigned to it (diamond-shaped targets) and then come back to the depot to reload its battery and start a second inspection route (circular-shape targets). Since UAVs make use of electric batteries with a limited driving range, there will be a maximum length set for each route. A cycle will be completed once all UAVs have returned to the depot after all target zones have been inspected.

Bad weather conditions and other factors might influence travel times, which also include monitoring times. For instance, under windy or rainy conditions, taking a good-quality picture might take longer than under good weather conditions. Thus, it will be assumed that traveling times are random variables following a best-fit probability distribution. Hence, our main goal will be to determine the set of sequential routes and assign the associated UAV-to-routes to minimize the expected total traveling time to complete an entire surveillance cycle. In our case, these routing plans will also have to satisfy a minimum reliability level. Indeed, a route failure will occur whenever the actual stochastic time employed by a UAV to complete a route exceeds the provided threshold time. Notice that, due to the stochastic behavior of travel times, this might happen even when the expected time for completing a route is lower than the threshold. Thus, the reliability level of each routing plan (i.e., the probability that the routing plan is free of failures) will also be computed since that might be of utmost importance for the decision-maker when choosing the ideal monitoring plan.

In order to solve the aforementioned stochastic surveillance VRP with maximum route length, we propose a methodology based on a simheuristic algorithm (Juan et al. 2018). Simheuristics combine 


\section{Panadero, Juan, Serrat, Grifoll, Dehghanimohamamdabadi, and Freixes}

heuristic algorithms with simulation, and have been employed to solve stochastic optimization problems in domain areas such as transportation (Guimarans et al. 2018), production scheduling (Hatami et al. 2018), patient appointment scheduling (Dehghanimohammadabadi et al. 2017), facility location (De Armas et al. 2017), telecommunication networks (Cabrera et al. 2014), or computational finance (Panadero et al. 2018).

The remaining sections of the paper are structured as follows: Section 2 briefly reviews related works; Section 3 provides a more detailed description of the surveillance VRP considered here; Section 4 describes the proposed simheuristic algorithm; Section 5 reports the results of a series of computational experiments; and finally, the main findings and future research lines are given in Section 6.

\section{RELATED WORK ON THE USE OF UAVS FOR SURVEILLANCE}

There has been a growing demand in using UAVs for surveillance and information collection tasks. Several applications include different environments and purposes with the common point of avoiding the need for direct human control, which is costly. In this sense, Semsch et al. (2009) address the problem of multi-UAV surveillance in complex urban environments with occlusions, minimizing the areas left uncovered by the UAVs. The task of search, exploration, and coverage also has been investigated by several authors. For instance, Nigam et al. (2012) includes flight test bed experiments in the control and coordination for persistent surveillance taking into account aircraft dynamic constrains such as refueling. Other applications include natural disaster scenarios surveillance (Neto et al. 2012), forest-fire surveillance (Alexis et al. 2009) or flood areas identification (Popescu et al. 2015).

Different initiatives have employed UAVs for maritime surveillance. Early applications consider data acquisition in specific campaigns in the field of the photogrammetry (Eisenbeiss 2004) or bathymetry (Wozencraft and Lillycrop 2003). Stacy et al. (2002) show UAV flight trials for maritime surveillance that employ sensors to detect and to classify vessels. Rubio et al. (2004) discuss search missions using UAVs to locate and track ocean debris. They used evolutionary computing algorithms for path planning, taking into account UAV performance degradation due to ice accumulation. Currently, more sophisticated sensors and new generation aerial vehicles have allowed to systematically obtain ocean parameters in open coastal areas, e.g., Schaub et al. (2018) or Reineman et al. (2016). Focusing on maritime surveillance, Bürkle and Essendorfer (2010) present the use of UAV monitoring and detecting people or vehicles attempting to enter into confined / protected areas in ports and coastlines. In similar terms, O'Young and Hubbard (2007) investigate cost-effective UAV technologies for maritime intelligence and surveillance. These applications combine together the use of UAVs with additional identification sensors, and prove the large demand of UAVs for different ranges of maritime surveillance. Despite several contributions (Rubio et al. 2004), UAV routing design still seems a challenge which deserves more attention in the field of maritime surveillance optimization. Fields related to rescue operations and search missions may be benefited by posing robust and efficient vehicle routing design for UAVs.

\section{ADDITIONAL PROBLEM DETAILS}

The UAV surveillance-routing problem with stochastic travel times and reliability-related probabilistic constraints is an extension of the deterministic VRP, which is a NP-hard problem (Toth and Vigo 2014). Let us consider a complete and undirected graph $G=(N, E)$, where: $(i) N=\{0,1, \ldots, n\}$ is a set of $n+1$ nodes including $n$ target zones as well as the depot (node 0 ); and (ii) $E=\{(i, j) / i, j \in N, i<j\}$ is the set of edges connecting the nodes. A fleet $V=\{1, \ldots, m\}$ of $m$ homogeneous UAVs, initially located at the depot, travel through the graph $G$ visiting all the target zones and then returning to the depot. Each UAV has a battery of limited duration, $t_{\max }>0$, which imposes a physical limit to the length of any route. Thus, UAVs might need to return to the depot for a reload before resuming a new route (multi-trip VRP). In our stochastic version, each edge $(i, j) \in E$ is associated with a random travel time, $T_{i j}>0$ (including the monitoring time), with $E\left[T_{i j}\right]<<t_{\max }$. This travel time is assumed to follow a best-fit probability distribution. Hence, the main goal is to find the surveillance-routing plan that minimizes the expected total 
time required to complete a full monitoring cycle. Notice that, while all target zones need to be visited during a surveillance cycle, visiting a target zone more than once inside each cycle will not pay off since it will exceed the cycle completion time. Also, since travel times are random, whenever a vehicle cannot complete the designed route in or before $t_{\max }$, a route failure occurs. This affects the reliability of the associated surveillance-routing plan, which is defined as the probability that the plan can be executed without any evident route failure. Analyzing reliability level of each surveillance-routing plan helps decision-makers to develop a trade-off mechanism between expected time and reliability when assessing a set of alternative plans.

Inside a cycle, each vehicle $v \in V$ can complete different routes (trips), denoted as: $r(v)=\left\{v_{1}, v_{2}, \ldots\right\}$. For each edge $(i, j) \in E$, consider the binary variable $x_{i j}^{v_{k}}$, which takes the value 1 if vehicle $v$ covers edge $(i, j)$ in trip $v_{k} \in r(v)$, and takes the value 0 otherwise. The condition that every target zone has to be visited exactly once inside a cycle, given a node $j \in N \backslash\{0\}$, can be expressed as:

$$
\sum_{v=1}^{m} \sum_{v_{k} \in r(v)} \sum_{(i, j) \in E} x_{i j}^{v_{k}}=1
$$

Similarly, given a vehicle $v \in V$, the total time employed by a route $v_{k} \in r(v)$ is given by:

$$
\sum_{(i, j) \in E} x_{i j}^{v_{k}} \cdot T_{i j}
$$

Hence, the following constraint should be satisfied $\forall v \in V$ and $\forall v_{k} \in r(v)$ :

$$
\sum_{(i, j) \in E} x_{i j}^{v_{k}} \cdot E\left[T_{i j}\right] \leq t_{\max }
$$

Notice that the total time necessary to complete a cycle can be computed as:

$$
\max _{v \in V}\left\{\sum_{v_{k} \in r(v)} \sum_{(i, j) \in E} x_{i j}^{v_{k}} \cdot T_{i j}\right\} \text {. }
$$

In order to minimize the expected value of expression (4), our task is to set the right values of the binary decision variables $x_{i j}^{v_{k}}$. Finally, since a surveillance-routing plan can be seen as a series system of each of its composing routes, the reliability-related probabilistic performance can be assessed through:

$$
\operatorname{Pr}\left(\sum_{(i, j) \in E} x_{i j}^{v_{k}} \cdot T_{i j}>t_{\text {max }}\right) \leq p^{*} \quad \forall v \in V, \quad \forall v_{k} \in r(v),
$$

where $p^{*}$ is a user-defined value in the $(0,1)$ interval.

\section{OVERVIEW OF OUR SIMHEURISTIC APPROACH}

Our solving approach relies on a simheuristic algorithm, which combines heuristic or metaheuristic algorithms with simulation (in any of its forms). These hybridization has been used to extend metaheuristic frameworks such as the greedy randomized adaptive search procedure (Ferone et al. 2018) or the iterated local search (Grasas et al. 2016). As any other simheuristic algorithm, the developed model in this study is composed of two different components: an optimization module which searches for promising solutions and a simulation model which assesses the promising solutions in a stochastic environment and might also guide the search process. In the optimization module, we use a multi-start framework to implement biased-randomization techniques within the constructive phase. The efficiency of these techniques combined with classical heuristics has been proved in some studies such as scheduling applications (Gonzalez-Neira et al. 2017) as well as vehicle routing problems (Dominguez et al. 2016; Martin et al. 2016). Figure 2 provides a flowchart overview of our simheuristic algorithm, which encompasses three stages: 


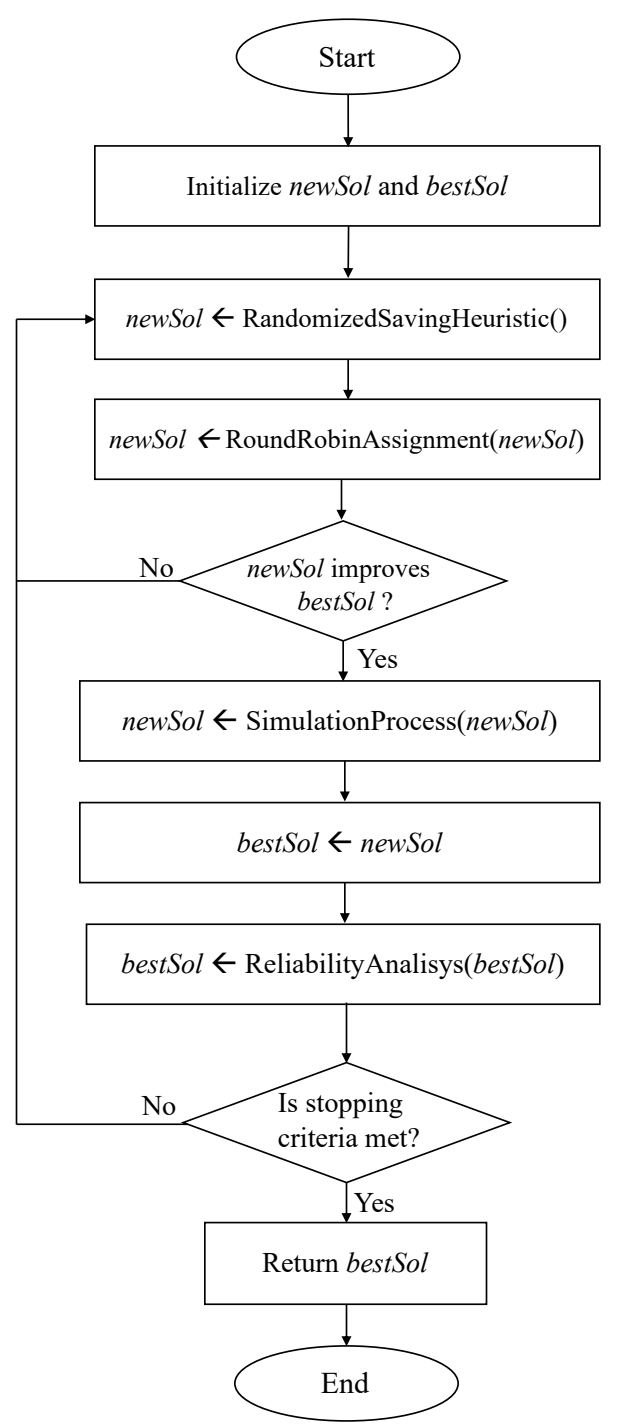

Figure 2: Flowchart of our simheuristic approach. 


\section{Panadero, Juan, Serrat, Grifoll, Dehghanimohamamdabadi, and Freixes}

- In the first step, an initial 'dummy' solution is constructed by generating one round-trip route from the depot to each target zone. Then, a route-merging process is started following a time-savings criterion. The time-based savings of merging any two routes is given by the savings in time associated with completing the merged route instead of the two original ones. The concept is used to generate a sorted list of potential merges, and these are completed following the corresponding order, from higher to lower savings. To satisfy the UAVs driving-range threshold, a potential merge can be completed only if the total time after the operation does not exceed the threshold. As a result of the merging process, a 'good' routing plan can be generated in just a few milliseconds (even for instances with several hundreds of target zones to inspect. The previous heuristic can be extended to an enhanced randomized algorithm (which usually provides a different routing plan each time it is run) by simply introducing biased randomization techniques (Grasas et al. 2017): with the use of a skewed probability distribution (the Geometric Distribution in our case), a descending probability of being selected can be assigned to each potential merge in the sorted list, which reduces the greedy behavior of the original heuristic without losing the logic behind it.

- In the second step, we employ a modified round-robin assignment process to distribute the routes generated in the previous step among the available UAVs. At the beginning, the $m$ most timeconsuming routes are assigned to the $m$ UAVs. Then, a first-in-first-out (FIFO) policy is applied, i.e., whenever a UAV arrives to the depot after completing a trip, it selects the most time-consuming route yet to be covered and starts a new trip. Again, the aforementioned process takes only a few milliseconds of computing time even for large-sized instances. As a result of this step, a new 'promising' surveillance-routing plan is generated.

- In the third step, a Monte Carlo simulation is run over the surveillance-routing plan proposed in the previous step. The simulation allows for obtaining point and interval estimates of several statistics of interests. In this work, we are especially interested in the expected completion time of the surveillance-routing plan when random travel times are considered as well as its associated reliability level.

- Finally, whenever the maximum computing time allowed has not been reached yet, the previous steps are repeated in order to generate as many promising surveillance-routing plans as possible and assess their quality via simulation.

\section{COMPUTATIONAL RESULTS}

Our simheuristic algorithm was implemented in Java code and run on a personal computer with 8 GB of RAM and an Intel Core i7 at $2.3 \mathrm{GHz}$. The number of simulations in each iteration of the simheuristic was setup to 5000 runs. Since there are no benchmark instances for the this vehicle surveillance problem with stochastic travel times and constrained driving ranges, we modified and extended a deterministic data set proposed by Augerat et al. (1995). Thus, the deterministic travel times were considered as mean values to generate the stochastic travel times. In our computational experiments, we have modeled the travel times $T_{i j}$ using Log-Normal probability distributions. The Log-Normal distribution is a more natural choice than the Normal distribution when modeling non-negative random variables, such as the elapsed time until an event occurs. Hence, it has been used to model variables such as the time it takes the vehicle to traverse a given edge (Gonzalez-Martin et al. 2018). In a real-world application, historical data could be used to model each $T_{i j}$ by a different probability distribution. The Log-Normal has two parameters, namely: the location parameter, $\mu$, and the scale parameter, $\sigma$. According to the properties of the Log-Normal distribution, these parameters will be given by the following expressions considering stochastic travel times between nodes $i$ and $j$ :

$$
\mu_{i j}=\ln \left(E\left[T_{i j}\right]\right)-\frac{1}{2} \ln \left(1+\frac{\operatorname{Var}\left[T_{i j}\right]}{E\left[T_{i j}\right]^{2}}\right)
$$




$$
\sigma_{i j}=\left|\sqrt{\ln \left(1+\frac{\operatorname{Var}\left[T_{i j}\right]}{E\left[T_{i j}\right]^{2}}\right)}\right| .
$$

In order to analyze the performance of the proposed simheuristic under different levels of variability, the scale parameter in the Log-Normal distributions for the edges travel times have been setup taking into account the relationship

$$
\operatorname{Var}\left[T_{i j}\right]=c E\left[T_{i j}\right]^{2}
$$

where $c=\exp \left(\sigma_{i j}^{2}\right)-1$, for three different values for the $c$ parameter, $c=0.25,0.5$, and 0.75 , respectively.

Table 1 summarizes the results obtained for the comparison of the simheuristic algorithm under deterministic and stochastic scenarios, for the mentioned three levels of variability. We have used nine instances with a diversity of number of nodes, travel times and topology of the graph. Number of UAVs (2) and number of routes per UAV (2) have been chosen as an illustrative case study.

For each instance we show: the Cycle Time of the Best Deterministic Solution (BDS), the threshold time $\left(t_{\max }\right)$, the Expected Cycle $(\mathrm{ECT})$ and the reliability $(\mathrm{RL})$ - i.e., the percentage of routes that are effectively completed without violating the $t_{\max }$ constraint in a stochastic environment - when the BDS is applied as a solution of the stochastic variant of the problem (OBS-D) and, the ECT and RL for Our Best Solution in a stochastic environment (OBS-S). Each instance has been computed considering the three different levels of variance (i.e., $c=0.25,0.5$ and 0.75 ). The reliability level has been empirically estimated using the equation (9).

$$
\text { Reliability }=1-\frac{\text { number of route failures }}{\text { number of simulation runs }} \text {. }
$$

After the simulation runs, as a sensitivity analysis, the simheuristic results state that:

a) The stochastic approach always performs better than the deterministic solution in a stochastic scenario, both from the point of view of the Expected Cycle Time, ECT, as well as the Level of Reliability, RL.

b) Higher variances implies higher ECT. Thus, the level of variance directly infers in a lower level of RL.

c) Despite we do not know a priori the exact effect of the threshold on the reliability of the resulting solution, we can infer that the dimension of the graph, as well as the existence of isolate nodes, sensibly affects the reliability level of the reported solution.

d) Regardless instances are not totally comparable, previous conclusions a) and b) can be extended in overall terms.

\section{CONCLUSIONS AND FUTURE WORK}

This paper presents a simheuristic algorithm to solve a stochastic version of the UAV surveillance-routing problem, where driving-range limitations are also considered. In this version of the problem, the main goal is to minimize the expected total time needed to complete a monitoring cycle using a limited fleet of UAVs. Since travel times are considered to be random variables, the designed surveillance-routing plans could suffer from route failures whenever the total time in covering a planned route exceeds a maximum driving range $t_{\max }$. Hence, the reliability value associated with each surveillance-routing plan is also a key performance indicator that needs to be taken into account. Overall reliability values are higher than $95 \%$, including the scenario with the highest variability $(c=0.75)$ what allows to validate the proposed simheuristic methodology.

Our algorithm combines a multi-start biased-randomized metaheuristic with Monte Carlo simulation. The simulation component is employed to assess the quality of promising surveillance-routing plans 
Panadero, Juan, Serrat, Grifoll, Dehghanimohamamdabadi, and Freixes

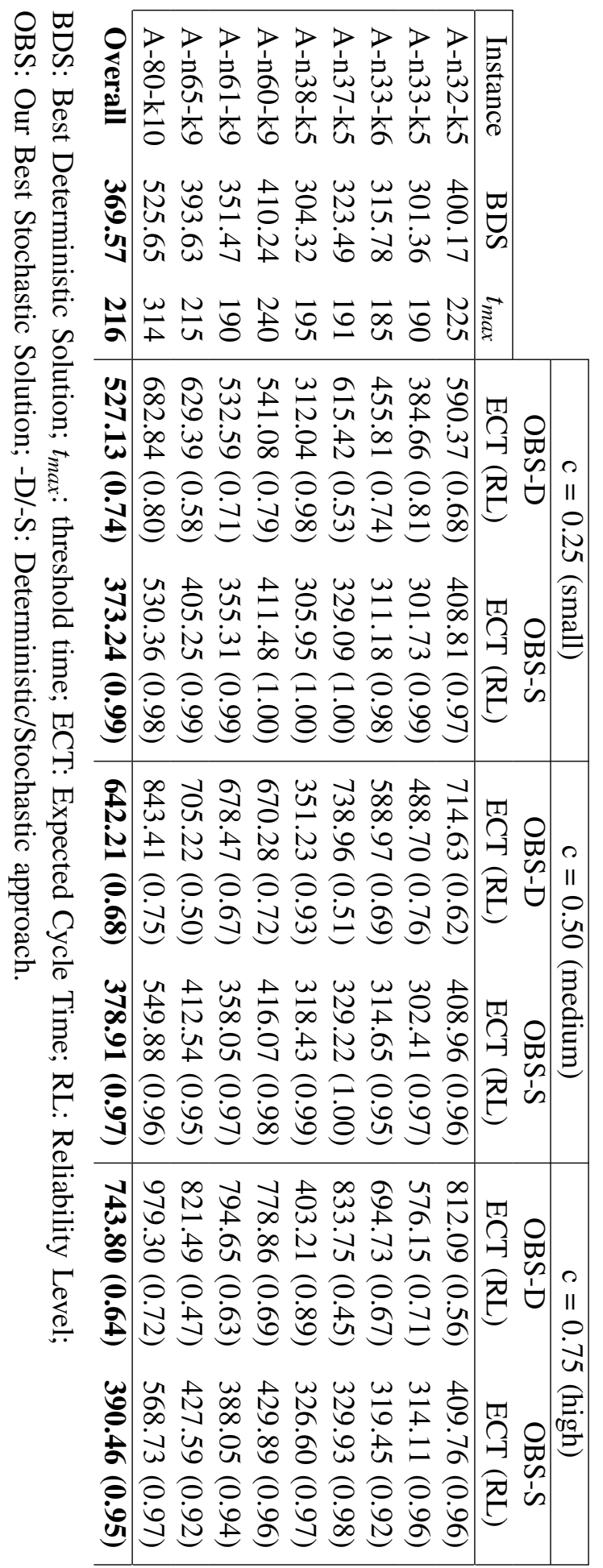




\section{Panadero, Juan, Serrat, Grifoll, Dehghanimohamamdabadi, and Freixes}

generated by the optimization component, both in terms of its expected completion time as well as in terms of its reliability level.

As future work, we plan to test our approach in more instances, including large-sized scenarios, as well as to perform a sensitivity analysis of the resulting optimized reliability levels under different values of the $t_{\max }$ parameter, for a given set of instances. It is also of our interest to include stochastic travel times depending on weather variables such as wind intensity and direction. Taking into account weather forecastings, this will fits with practical implementations of UAVs routing in problems in mass rescue operations or debris tracking in the framework of maritime surveillance. Also, the proposed algorithm could be extended to include sustainability indicators that provide a richer evaluation of the routing plans.

On the other hand, the algorithm described in this paper can be enhanced in several directions: (i) instead of using a simple multi-start framework, a metaheuristic one could be employed in order to better guide the search process if more computing time is available, as done in De Armas et al. (2017); (ii) in the third step of the algorithm, simulations with a reduced number of runs could be employed so a set of 'elite' surveillance-routing plans could be selected in a short computing time; statistics on each of these plans can then be accurately provided by employing an additional step in which longer simulations are run; and (iii) a 'safety stock' $\varepsilon>0$ could be used during the route-merging process to increase the reliability of the routing plans, i.e.: instead of considering $t_{\max }$ as a threshold, a limit given by $t_{\max }-\varepsilon$ could be used during the merging process to build shorter and more reliable routes. Notice that in some cases this could also be useful to better distribute the monitoring load among the fleet of UAVs.

\section{ACKNOWLEDGEMENTS}

This work has been partially supported by the Catalan Agency for Management of University and Research Grants (2017-DI-066), the Spanish Ministry of Economy and Competitiveness / FEDER (MTM2015-64465C2-1-R), and the Erasmus+ program (2018-1-ES01-KA103-049767).

\section{REFERENCES}

Alexis, K., G. Nikolakopoulos, A. Tzes, and L. Dritsas. 2009. "Coordination of Helicopter UAVs for Aerial Forest-Fire Surveillance". Applications of Intelligent Control to Engineering Systems 39:169-193.

Augerat, P., J. M. Belenguer, E. Benavent, A. Corberan, D. Naddef, and G. Rinaldi. 1995. "Computational Results with a Branch-and-Cut Code for the Capacitated Vehicle Routing Problem”. Technical Report 949-M, Universit Joseph Fourier, Grenoble, France.

Bürkle, A. and B. Essendorfer. 2010. "Maritime Surveillance with Integrated Systems". In 2010 International WaterSide Security Conference, 1-8, doi:10.1109/WSSC.2010.5730231. Piscataway, New Jersey: Institute of Electrical and Electronics Engineers, Inc.

Cabrera, G., A. A. Juan, D. Lázaro, J. M. Marquès, and I. Proskurnia. 2014. "A Simulation-Optimization Approach to Deploy Internet Services in Large-Scale Systems with User-Provided Resources". Simulation 90(6):644-659.

De Armas, J., A. A. Juan, J. M. Marquès, and J. P. Pedroso. 2017. "Solving the Deterministic and Stochastic Uncapacitated Facility Location Problem: from a Heuristic to a Simheuristic". Journal of the Operational Research Society 68(10):1161-1176.

Dehghanimohammadabadi, M., M. Rezaeiahari, and T. K. Keyser. 2017. "Simheuristic of Patient Scheduling using a TableExperiment Approach: Simio and Matlab Integration Application". In Proceedings of the 2017 Winter Simulation Conference, edited by W. K. V. Chan, A. D’Ambrogio, G. Zacharewicz, N. Mustafee, G. Wainer, and E. Page, 2929-2939. Piscataway, New Jersey: Institute of Electrical and Electronics Engineers, Inc.

Dominguez, O., D. Guimarans, A. A. Juan, and I. de la Nuez. 2016. "A Biased-Randomised Large Neighbourhood Search for the Two-Dimensional Vehicle Routing Problem with Backhauls”. European Journal of Operational Research 255(2):442-462.

Eisenbeiss, H. 2004. "A Mini Unmanned Aerial Vehicle (UAV): System Overview and Image Acquisition". International Archives of Photogrammetry. Remote Sensing and Spatial Information Sciences 36(5/W1):1-7.

Faulin, J., M. Gilibert, A. A. Juan, X. Vilajosana, and R. Ruiz. 2008. "SR-1: A Simulation-based Algorithm for the Capacitated Vehicle Routing Problem”. In Proceedings of the 2008 Winter Simulation Conference, edited by S. J. Mason, R. R. Hill, L. Moench, O. Rose, T. Jefferson, and J. W. Fowler, 2708-2716. Piscataway, New Jersey: Institute of Electrical and Electronics Engineers, Inc.

Faulin, J. and A. A. Juan. 2008. "The ALGACEA-1 Method for the Capacitated Vehicle Routing Problem". International Transactions in Operational Research 15(5):599-621. 


\section{Panadero, Juan, Serrat, Grifoll, Dehghanimohamamdabadi, and Freixes}

Ferone, D., A. Gruler, P. Festa, and A. A. Juan. 2018. "Enhancing and Extending the Classical GRASP Framework with Biased Randomisation and Simulation". Journal of the Operational Research Society:1-14, doi:10.1080/01605682.2018.1494527.

Gonzalez-Martin, S., A. A. Juan, D. Riera, M. G. Elizondo, and J. J. Ramos. 2018. "A Simheuristic Algorithm for Solving the Arc Routing Problem with Stochastic Demands". Journal of Simulation 12(1):53-66.

Gonzalez-Neira, E. M., D. Ferone, S. Hatami, and A. A. Juan. 2017. "A Biased-Randomized Simheuristic for the Distributed Assembly Permutation Flowshop Problem with Stochastic Processing Times". Simulation Modelling Practice and Theory 79:23-36.

Grasas, A., A. A. Juan, J. Faulin, J. de Armas, and H. Ramalhinho. 2017. "Biased Randomization of Heuristics Using Skewed Probability Distributions: a Survey and Some Applications”. Computers \& Industrial Engineering 110:216-228.

Grasas, A., A. A. Juan, and H. R. Lourenço. 2016. "SimILS: a Simulation-based Extension of the Iterated Local Search Metaheuristic for Stochastic Combinatorial Optimization”. Journal of Simulation 10(1):69-77.

Guimarans, D., O. Dominguez, J. Panadero, and A. A. Juan. 2018. "A Simheuristic Approach for the TwoDimensional Vehicle Routing Problem with Stochastic Travel Times”. Simulation Modelling Practice and Theory 89:114,doi:10.1016/j.simpat.2018.09.004.

Hatami, S., L. Calvet, V. Fernández-Viagas, J. M. Framiñán, and A. A. Juan. 2018. "A Simheuristic Algorithm to Set Up Starting Times in the Stochastic Parallel Flowshop Problem". Simulation Modelling Practice and Theory 86:55-71.

Juan, A. A., J. Faulin, R. Ruiz, B. Barrios, M. Gilibert, and X. Vilajosana. 2009. "Using Oriented Random Search to Provide a Set of Alternative Solutions to the Capacitated Vehicle Routing Problem". In Operations Research and Cyber-Infrastructure, 331-345. Springer.

Juan, A. A., W. D. Kelton, C. S. Currie, and J. Faulin. 2018. "Simheuristics Applications: Dealing with Uncertainty in Logistics, Transportation, and other Supply Chain Areas". In Proceedings of the 2018 Winter Simulation Conference, edited by M. Rabe, A. A. Juan, N. Mustafee, A. Skoogh, S. Jain, and B. Johansson, 3048-3059. Piscataway, New Jersey: Institute of Electrical and Electronics Engineers, Inc.

Martin, S., D. Ouelhadj, P. Beullens, E. Ozcan, A. A. Juan, and E. K. Burke. 2016. "A Multi-Agent Based Cooperative Approach to Scheduling and Routing". European Journal of Operational Research 254(1):169-178.

Neto, J. M. M., R. A. da Paixão, L. R. L. Rodrigues, E. M. Moreira, J. C. J. dos Santos, and P. F. F. Rosa. 2012 . "A Surveillance Task for a UAV in a Natural Disaster Scenario". In 2012 IEEE International Symposium on Industrial Electronics, 1516-1522, doi:10.1109/ISIE.2012.6237316. Piscataway, New Jersey: Institute of Electrical and Electronics Engineers, Inc.

Nigam, N., S. Bieniawski, I. Kroo, and J. Vian. 2012. "Control of Multiple UAVs for Persistent Surveillance: Algorithm and Flight Test Results". IEEE Transactions on Control Systems Technology 20(5):1236-1251.

O'Young, S. and P. Hubbard. 2007. "RAVEN: A Maritime Surveillance Project using Small UAV". In 2007 IEEE Conference on Emerging Technologies and Factory Automation (EFTA 2007), 904-907, doi:10.1109/EFTA.2007.4416878. Piscataway, New Jersey: Institute of Electrical and Electronics Engineers, Inc.

Panadero, J., J. Doering, R. Kizys, A. A. Juan, and A. Fito. 2018. "A Variable Neighborhood Search Simheuristic for Project Portfolio Selection under Uncertainty". Journal of Heuristics:1-23, doi:10.1007/s10732-018-9367-z.

Popescu, D., L. Ichim, and T. Caramihale. 2015. "Flood Areas Detection based on UAV Surveillance System". In 2015 19th International Conference on System Theory, Control and Computing (ICSTCC), 753-758, doi:10.1109/ICSTCC.2015.7321384. Piscataway, New Jersey: Institute of Electrical and Electronics Engineers, Inc.

Reineman, B. D., L. Lenain, and W. K. Melville. 2016. "The Use of Ship-Launched Fixed-Wing UAVs for Measuring the Marine Atmospheric Boundary Layer and Ocean Surface Processes”. Journal of Atmospheric and Oceanic Technology 33(9):20292052.

Rubio, J. C., J. Vagners, and R. Rysdyk. 2004. "Adaptive Path Planning for Autonomous UAV Oceanic Search Missions”. In AIAA 1st Intelligent Systems Technical Conference, Number 6228, 1-10, doi:10.2514/6.2004-6228. Reston, Vancouver: AIAA.

Schaub, J., B. P. Hunt, E. A. Pakhomov, K. Holmes, Y. Lu, and L. Quayle. 2018. "Using Unmanned Aerial Vehicles (UAVs) to Measure Jellyfish Aggregations". Marine Ecology Progress Series 591:29-36.

Semsch, E., M. Jakob, D. Pavlicek, and M. Pechoucek. 2009. "Autonomous UAV Surveillance in Complex Urban Environments". In Proceedings of the 2009 IEEE/WIC/ACM International Joint Conference on Web Intelligence and Intelligent Agent Technology, Volume 2, 82-85, doi:10.1109/WI-IAT.2009.132. Piscataway, New Jersey: Institute of Electrical and Electronics Engineers, Inc.

Stacy, N., D. Craig, J. Staromlynska, and R. Smith. 2002. "The Global Hawk UAV Australian Deployment: Imaging Radar Sensor Modifications and Employment for Maritime Surveillance”. In IEEE International Geoscience and Remote Sensing Symposium, Volume 2, 699-701, doi:10.1109/IGARSS.2002.1025623. Piscataway, New Jersey: Institute of Electrical and Electronics Engineers, Inc.

Toth, P. and D. Vigo. 2014. Vehicle Routing: Problems, Methods, and Applications. Philadelphia, PA: Society for Industrial and Applied Mathematics. 


\section{Panadero, Juan, Serrat, Grifoll, Dehghanimohamamdabadi, and Freixes}

Wozencraft, J. M. and W. J. Lillycrop. 2003. "SHOALS Airborne Coastal Mapping: Past, Present, and Future". Journal of Coastal Research 38:207-215.

\section{AUTHOR BIOGRAPHIES}

JAVIER PANADERO is an Assistant Professor of Simulation and High Performance Computing in the Computer Science, Multimedia and Telecommunication Department at the Universitat Oberta de Catalunya (Barcelona, Spain). He is also a Lecturer at the Euncet Bussines School, and a member of the ICSO@IN3 research group. He holds a Ph.D. and a M.S. in Computer Science. His major research areas are: high performance computing, modeling and analysis of parallel applications, and simheuristics. He has co-authored more than 18 articles published in journals and conference proceedings. His website address is http://www.javierpanadero.com and his email address is jpanaderom@uoc.edu.

ANGEL A. JUAN is a Full Professor of Operations Research \& Industrial Engineering in the Computer Science Dept. at the Universitat Oberta de Catalunya (Barcelona, Spain). He is also the Director of the ICSO research group at the Internet Interdisciplinary Institute and Lecturer at the Euncet Business School. Dr. Juan holds a Ph.D. in Industrial Engineering and an M.Sc. in Mathematics. He completed a predoctoral internship at Harvard University and postdoctoral internships at Massachusetts Institute of Technology and Georgia Institute of Technology. His main research interests include applications of simheuristics and learnheuristics in computational logistics and finance, as well as mathematical e-learning. He has published about 80 articles in JCR-indexed journals and nearly 200 papers indexed in Scopus. His website address is http://ajuanp.wordpress.com and his email address is ajuanp@uoc.edu.

CARLES SERRAT is an Associate Professor at the Department of Mathematics at the Universitat Politècnica de CatalunyaBarcelonaTECH, at the Barcelona School of Building Construction (Catalonia, Spain). His areas or research include, but are not limited to, methodological and applied statistics as well as methaheuristics to fields like public health, construction, civil engineering, economy, logistics, and transport. Specifically he focuses on approaches based on survival analysis techniques, longitudinal data analysis, and missing data analysis. Professor Serrat has been granted for visiting scholarships at Harvard University and Hasselt University and visiting researcher stays at Open University of Catalonia, Trinity College Dublin, Universidad Nacional de Colombia, and Universidad de La Sabana. His website address is http://cserrat.wordpress.com/ and his email address is carles.serrat@upc.edu.

MANEL GRIFOLL is a Lecturer of the Department of Civil and Environmental Engineering of the Universitat Politecnica de Catalunya (UPC-BarcelonaTECH). He holds a Ph.D. and a M.S. in Civil Engineering. His main research interest include multi-port traffic analysis, weather ship routing and applied marine science. He has published more than 35 articles in JCRindexed journals and more than 40 documents indexed in Scopus. Currently, he is the vice-dean of International Relations and Research of the Barcelona School of Nautical Studies of the UPC-BarcelonaTECH. His e-mail address is manel.grifoll@upc.edu.

MOHAMMAD DEHGHANIMOHAMMADABADI is an Assistant Teaching Professor of Mechanical and Industrial engineering at Northeastern University (Boston, USA). He received his Ph.D. in Engineering Management from Western New England University (USA) in 2016. His research is mainly focused on developing and generalizing simulation and optimization frameworks in difference disciplines such as healthcare, supply chain, and manufacturing. His website address is http://www.mie.neu.edu/people/dehghani-mohammad and his e-mail address is m.dehghani@northeastern.edu.

ALFONS FREIXES is the Director of the Euncet Business School (Barcelona, Spain). He holds a M.Sc. in Management of Information Technologies from La Salle - Ramon Llull University, and a B.S. in Industrial Engineering from the Universitat Politècnica de Catalunya-BarcelonaTECH. He is completing a Ph.D. in the AGAUR Industrial Doctorate Program at the Universitat Oberta de Catalunya. His research area focuses on applications of statistical, computational, and IoT-related methods in smart and sustainable cities, including efficient coordination of unmanned aerial vehicles. His email address is afreixes@euncet.es. 